\title{
SÍNDROME INFLAMATÓRIA MULTISSISTÊMICA PEDIÁTRICA ASSOCIADA AO COVID-19
}

\section{MULTISISTEMIC PEDIATRIC INFLAMMATORY SYNDROME ASSOCIATED TO COVID-19}

\author{
Gabriele Maria Viana Martins' ${ }^{1}$, Suellen dos Santos Rangel', Mariana Pompermayer Eduardo', Inês Raquel Alves \\ da Silva Rosário', Eliane Cristina Casimiro Alves Dias² \\ ${ }^{1}$ Acadêmicas em Medicina da Faculdade de Medicina de Campos, Campos dos Goytacazes-RJ. \\ 2 Professora de Pediatria da Faculdade de Medicina de Campos, Campos dos Goytacazes-RJ.
}

*Autora de Contato: Gabriele Maria Viana Martins, (22) 99903-5315; gabimariavianam@gmail.com

\section{RESUMO}

O objetivo deste trabalho é conhecer a Síndrome Inflamatória Multissistêmica Pediátrica (SIM-P) associada à COVID-19 e alertar profissionais de saúde sobre a importância do diagnóstico e condutas precoces. Esse estudo trata-se de uma revisão integrativa de literatura, utilizando artigos nacionais e internacionais dos seguintes bancos de dados: PUBMED, SciELO e Clinicalkey. Sabe-se que a COVID19 é uma doença altamente contagiosa que rapidamente se tornou um problema de saúde pública mundial. Desde o início da pandemia, os idosos e portadores de comorbidades fazem parte do grupo de risco. Enquanto, as crianças, normalmente, são assintomáticas e preocupam mais pelo fato serem consideradas um reservatório do vírus, podendo transmiti-lo. No entanto, recentemente, foram notificados casos infantis que tiveram uma evolução atípica. A SIM-P associada à COVID-19 foi relatada pela primeira vez na Europa como uma doença que ocorre semanas após a fase aguda da infecção pelo SARS-CoV2. As manifestações clínicas e as características laboratoriais dessa doença são semelhantes a outras doenças inflamatórias presentes na pediatria como a síndrome de Kawasaki típica, Kawasaki incompleta, síndrome do choque tóxico, sepse bacteriana e síndrome de ativação macrofágica. Diferentemente do que é observado nas demais doenças inflamatórias, a SIM-P afeta preferencialmente crianças maiores que 5 anos, afrodescendentes, além de apresentar uma maior incidência de disfunção cardíaca. 0 tratamento deve envolver ações multidisciplinares e ser precoce a fim de minimizar as complicações associadas a essa patologia, principalmente as cardíacas e, assim, diminuir a mortalidade.

Palavras Chaves: Coronavírus; Síndrome Inflamatória; Pediatria.
ABSTRACT

The purpose of this review is to elucidate MultiSystem Inflammatory Syndrome in Children (MIS-C) associated with COVID-19 and alert health care professionals about the importance of an early diagnosis and conduct. This study is an integrated literature review, using national and international articles from the following databases: PUBMED, SciELO and Clinicalkey. COVID-19 is known to be a highly contagious disease that has quickly become a worldwide public health problem. Since the beginning of the pandemic, the elderly and those with comorbidities have been a part of the risk group. Children are usually asymptomatic and cause more concern due to them being potential carriers of the virus, with high chances of transmitting it. However, childhood cases with an atypical evolution have recently been reported. MIS-C associated with COVID19 was first reported in Europe as a disease that occurs weeks after the acute phase of the SARS-CoV-2 infection. The clinical manifestations and laboratory characteristics of this disease are similar to other inflammatory diseases present in pediatrics such as the typical Kawasaki syndrome, incomplete Kawasaki, Toxic shock syndrome, bacterial sepsis and Macrophage activation syndrome. Unlike what is observed in other inflammatory diseases, MIS-C preferentially affects children older than 5 years, of African descent, in addition to being related to a higher incidence of cardiac dysfunction. Treatment must be early and involve multidisciplinary actions in order to minimize complications associated with this pathology, especially cardiac complications and, thus, reduce mortality.

KeyWords: Coronavirus; Inflammatory Syndrome; Pediatrics. 


\section{INTRODUÇÃO}

Em 2019 em Wuhan, na China, surgiu um novo coronavírus denominado SARS-CoV-2 ("severe acute respiratory syndrome coronavirus-2") que é o causador da COVID-19 ("coronavirus disease2019"), doença que tornou-se um problema de saúde pública mundial e, portanto, foi declarada pela Organização Mundial de Saúde (OMS) como pandemia em março de 2020 (SOCIEDADE BRASILEIRA DE REUMATOLOGIA, 2020).

ssa patologia pode acometer indivíduos de qualquer faixa etária. Os idosos acima de 60 anos e portadores de doenças não transmissíveis subjacentes como hipertensão arterial sistêmica, doença cardíaca, doença pulmonar crônica e neoplasias apresentam fatores de risco para o desenvolvimento da forma grave da doença (WHO, 2020).

Por outro lado, as crianças e adolescentes, na maioria das vezes, representam uma pequena porção de casos de COVID-19 e quando infectados pelo SARS-CoV-2 são assintomáticas ou apresentam a forma leve da doença. Apesar de raros os óbitos, a vulnerabilidade das crianças é crescente, podendo evoluir com manifestações clínicas graves associadas à COVID-19 que são englobadas na Síndrome Inflamatória Multissistêmica Pediátrica (SIM-P) (SOCIEDADE BRASILEIRA DE PEDIATRIA, 2020).

O primeiro relato da SIM-P ocorreu em abril de 2020 pela Sociedade de Pediatria do Reino Unido que emitiu um alerta sobre uma nova apresentação clínica observada em crianças na faixa etária de 4-17 anos. Posteriormente, outros países descreveram casos de pacientes pediátricos que foram internados por desenvolver uma inflamação multissistêmica semanas após a fase aguda da COVID-19 (WHO, 2020).

A maioria das crianças diagnosticadas com SIM-P apresentaram testes por reação em cadeia de polimerase em tempo real (RT-PCR) ou sorológico positivo para o SARS-CoV-2, o que sugere uma associação entre as doenças. No entanto, a ausência dos exames positivos não deve excluir a hipótese diagnóstica visto que a história epidemiológica também deve ser considerada (CDC, 2020).

Esse trabalho tem como objetivo conhecer a Síndrome Inflamatória Multissistêmica Pediátrica associada à COVID-19 e alertar profissionais de saúde a respeito da importância do diagnóstico e conduta dessa patologia que está em ascensão.
MÉTODOS

O presente estudo é uma revisão bibliográfica sobre a Síndrome Inflamatória Multissistêmica Pediátrica associada à COVID-19, realizada nos meses de agosto e setembro de 2020. Foram realizadas consultas ao banco de dados do PUBMED, SciELO e Clinicalkey, sendo incluídos artigos publicados na íntegra, em português ou inglês. As palavras-chave utilizadas na procura foram "Coronavírus", "Síndrome Inflamatória" e "Pediatria". Após pesquisa, buscou-se compreender os principais parâmetros relatados até o momento sobre essa patologia e relatá-los.

\section{CLÍNICA E DIAGNÓTICO}

A Síndrome Inflamatória Multissistêmica Pediátrica associada à COVID-19 é rara e apresenta características comuns com outras doenças pediátricas, consideradas diagnósticos diferenciais da SIM-P (Tabela 1). Destacam-se a doença de Kawasaki e as síndromes de choque tóxico estafilocócico e estreptocócico (SOCIEDADE BRASILEIRA DE PEDIATRIA, 2020).

\section{Tabela 1: DIAGNÓSTICOS DIFERENCIAIS}

Sepse bacteriana;

Doença de Kawasaki;

Síndrome da pele escaldada;

Síndrome do choque tóxico;

Apendicite;

Síndrome de ativação macrofágica;

Lúpus eritematoso sistêmico juvenil;

Vasculites primárias;

Infecções virais: Dengue, Epstein-Barr, Citomegalovírus, Adenovirus e Enterovirus*.

\section{*A pesquisa desses virus deve ser ponderada na investigação diagnóstica. \\ Fonte adaptada de (SOCIEDADE BRASILEIRA \\ DE PEDIATRIA, 2020, p. 4).}

A doença de Kawasaki é uma vasculite sistêmica pediátrica que apresenta uma maior predileção por meninos menores de 5 anos. Acomete, preferencialmente, artérias de pequeno ou médio calibre e a principal complicação é aneurisma das artérias coronárias, sendo importante 
em todos os casos em que suspeita-se da doença a realização do ecocardiograma (VINER et al, 2020). A comparação da SIM-P com a doença de Kawasaki acontece, justamente, porque ambas danificam vasos sanguíneos e podem cursar com choque tóxico estafilocócico e estreptocócico, além de apresentar características clínicas e laboratoriais semelhantes (CAMPOS et al, 2020).

O quadro clínico apresentado pelos pacientes pediátricos com SIM-P é amplo e os sintomas mais comuns são a febre, que se caracteriza por ser alta e persistente e as manifestações gastrointestinais, como vômito, diarreia e dor abdominal intensa. Além disso, podem ser observados conjuntivite não purulenta, edema de mãos e pés, exantema pleomórfico, I infadenopatia ge nera lizada, hepatoesplenomegalia, pleurite, pericardite, ascite, cefaleia, irritabilidade, alteração de nível de consciência e aneurisma de artérias coronárias, podendo complicar em choque cardiogênico. Inicialmente, os sintomas respiratórios não estão presentes, porém com a evolução da doença podem aparecer (PANUPATTANAPONG; BROOKS, 2020).

Tais manifestações clínicas mostram-se equivalentes à doença de Kawasaki a qual observase obrigatoriamente febre alta por pelo menos 5 dias associada a 4 ou 5 dos seguintes critérios: exantema polimorfo, hiperemia conjuntival, alterações de cavidade oral e lábios, alterações de extremidades e linfadenopatia cervical aguda $\geq 1,5 \mathrm{~cm}$ normalmente unilateral (MCCRINDLE, 2017).

No entanto, existem características que permitem a diferenciação entre as doenças. Ao contrário da doença de Kawasaki, a SIM-P costuma acometer crianças maiores de 5 anos, apresenta maiores taxas de envolvimento cardíaco e apresenta um predomínio por afrodescendentes (CAMPOS et al, 2020).

As alterações laboratoriais da SIM-P se caracterizam por aumento dos marcadores inflamatórios e de função miocárdica, ou seja, observam-se valores elevados de proteína $\mathrm{C}$ reativa (PCR), velocidade de hemossedimentação (VHS), ferritina, procalcitonina, triglicérides e D-dímero. Além disso, esses pacientes podem apresentar alterações hematológicas como anemia, linfopenia, leucopenia, trombocitopenia e coagulopatia de consumo (SOCIEDADE BRASILEIRA DE PEDIATRIA, 2020).

Em casos avançados, complicações como disfunção miocárdica e diminuição da resistência vascular periférica podem resultar em sinais de choque com hipotensão, taquicardia e distúrbio de perfusão os quais necessitam, na maioria das vezes, de ressuscitação cardiorrespiratória. Outras complicações que demandam atenção são miocardite, aneurisma coronariano gigante e lesão renal aguda. Na maioria das complicações, as internações na unidade de terapia intensiva são imprescindíveis (CDC, 2020).

Crianças e adolescentes com sinais e sintomas característicos da SIM-P que apresentem história epidemiológica sugestiva de infecção pelo SARS-CoV-2 devem realizar o RT-PCR, sorologia IgM e IgG ou pesquisa de antígeno. Além disso, é necessário dosar a atividade inflamatória e investigar órgãos e sistemas acometidos (CDC, 2020).

Inicialmente, é de grande importância monitorar os marcadores inflamatórios, tais como, PCR e VHS. Ainda se faz necessário solicitar hemograma, ureia e creatinina para avaliação da função renal, glicemia, eletrólitos e provas de função hepática e de vias biliares. Após afastamento de outras causas infecciosas e confirmação do quadro, acrescenta-se a avaliação inicial a dosagem de LDH, triglicerídeos, Creatina kinase (CK), ferritina, TAP, PTT, D-dímero, fibrinogênio (SOCIEDADE BRASILEIRA DE REUMATOLOGIA, 2020).

Para avaliar a função cardíaca dosa-se a troponina, $\mathrm{CK}-\mathrm{MB}$, mioglobina, pró-BNP e solicita-se os exames eletrocardiograma e ecocardiograma (SOCIEDADE BRASILEIRA DE REUMATOLOGIA, 2020). Já a tomografia computadorizada ou ressonância nuclear magnética são importantes em casos suspeitos de aneurisma coronariano, assim como para fazer acompanhamento do mesmo (RAMCHARAN et al, 2020).

Em casos de sinais e sintomas respiratórios faz-se necessário avaliar o tórax pelos exames de imagem e solicitar gasometria arterial. Todavia, nos casos de sintomatologia gastrointestinal é indispensável a realização de exames de imagem abdominal. A procalcitonina e o painel de citocinas devem ser solicitados para acompanhamento do quadro inflamatório, se disponíveis (CAMPOS et al, 2020).

Para auxílio no diagnóstico dessa síndrome, até o momento, foram estabelecidos critérios pelo Centers for Disease Control (CDC) e pela OMS, os quais estão descritos na tabela 2. 


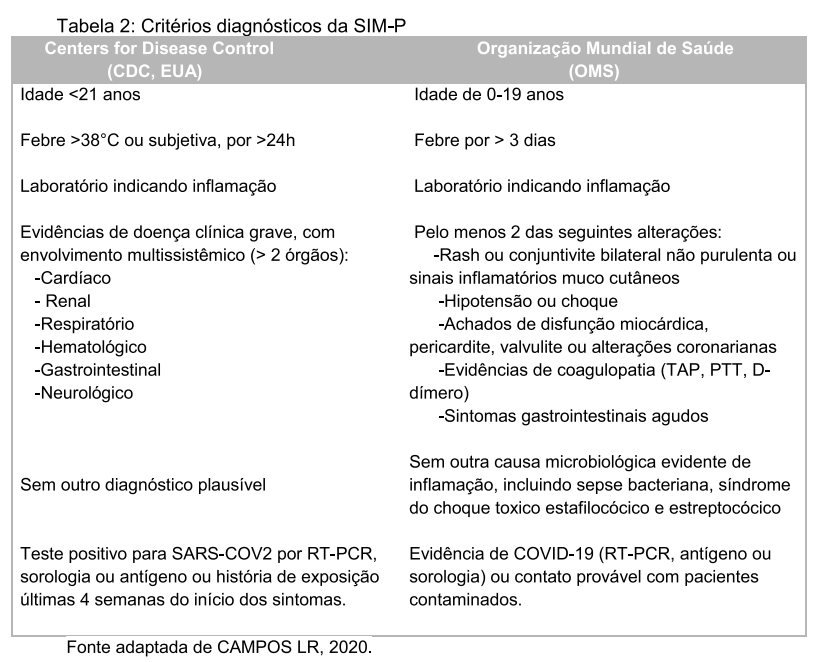

\section{PRINCÍPIOS DO TRATAMENTO}

Ações conjuntas de pediatras, infectologistas, reumatologista, cardiologista e intensivista devem, sempre que possível, serem solicitadas de forma precoce para garantir um tratamento imediato, com o objetivo de diminuir o estado inflamatório sistêmico e restabelecer o funcionamento adequado dos órgãos e sistemas, a fim de minimizar a incidência de sequelas como lesões coronarianas e disfunção cardíaca, além de diminuir a mortalidade (SOCIEDADE BRASILEIRA DE PEDIATRIA, 2020).

Crianças com as manifestações clínicas características da SIM-P devem ser tratadas como suspeitas de COVID-19, seguindo os protocolos locais de manejo e antibioticoterapia empírica. Em casos de SIM-P leve ou moderada, está indicado o tratamento de suporte que, normalmente, é suficiente para esses pacientes. Se houver deterioração clínica ou doença severa, é preciso analisar transferência para terapia intensiva pediátrica, devido à alta frequência de choque (ROYAL COLLEGE OF PAEDIATRICS AND CHILD HEALTH et al., 2020, p. 3).

O uso do corticoide deve ser considerado em pacientes com comprometimento miocárdico, mesmo que ainda mínimo. Nos casos leves, pode ser indicada a dose de $2 \mathrm{mg} / \mathrm{kg} / \mathrm{dia}$ de metilprednisolona 4 vezes com redução progressiva em aproximadamente 2 semanas. Para os casos mais graves, é indicado o uso de metilprednisolona em forma de pulsoterapia na dose de $30 \mathrm{mg} / \mathrm{kg} / \mathrm{dia}$ por 3 dias, e nos casos moderados $10-20 \mathrm{mg} / \mathrm{kg} /$ dia por 1 3 dias. Após período de pulsoterapia, deve-se prescrever uma dose de manutenção de $2 \mathrm{mg} /$ $\mathrm{kg} /$ dia (SOCIEDADE BRASILEIRA DE PEDIATRIA, 2020).

Pacientes com SIM-P têm uma tendência maior de apresentar fenômenos tromboembólicos e a dosagem do D-dímero costuma ser muito elevada, no entanto, o uso de anticoagulante ainda é controverso. Alguns casos de SIM-P leves a moderados podem ser manejados com dose profilática de enoxaparina e, casos mais graves, com dose terapêutica. $\mathrm{O}$ uso do anticoagulante deve ser analisado individualmente, levando em consideração o risco de sangramento do paciente (CAMPOS et al, 2020).

Em relação às terapias antivirais e imunomoduladoras, essas só devem ser consideradas no âmbito de protocolos clínicos e necessitam ser discutidas com os comitês de ética locais (SOCIEDADE BRASILEIRA DE PEDIATRIA, 2020).

O uso da imunoglobulina endovenosa (IGEV) deve ser considerado nos casos moderados e graves e nos pacientes que se enquadram nos critérios completos ou parciais para a síndrome de Kawasaki e/ou síndrome de ativação macrofágica. Pode-se considerar também seu uso na síndrome do choque tóxico refratária ao tratamento convencional (CAMPOS et al, 2020).

Os imunomoduladores devem ser usados somente nos casos refratários ao tratamento com IGEV e pulsoterapia com metilprednisolona, já que, nesses casos, pode estar acontecendo uma liberação exacerbada de citocinas. (RAMCHARAN et al, 2020).

As crianças com SIM-P costumam apresentar uma evolução favorável e após alta hospitalar, os pacientes precisam ser acompanhados, especialmente, em casos de complicações cardíacas como aneurismas coronarianos e miocardiopatia, além de pneumopatias, doença renal aguda, tromboses e neuropatias (SOCIEDADE BRASILEIRA DE PEDIATRIA, 2020).

\section{CONSIDERAÇÕES FINAIS}

Com este trabalho, conclui-se que a SIM-P associada à COVID-19 não se apresenta muito bem elucidada, com quadro clínico amplo, e características comuns a outras doenças inflamatórias, principalmente a doença de Kawasaki. No entanto, através da sintomatologia e marcadores inflamatórios associados à história de infecção pelo SARS-CoV-2 é possível fazer o diagnóstico.

Uma vez diagnosticado, é imprescindível o 
início do tratamento de forma precoce para evitar possíveis alterações que comprometam órgãos e sistemas. Ações conjuntas de pediatras, infectologista, reumatologista, cardiologista e intensivista precisam ser solicitadas rapidamente para garantir um tratamento eficaz, a fim de evitar complicações, como a disfunção cardíaca, por exemplo, visto que esta pode resultar em choque.
Conforme exposto, embora se trate de uma patologia nova, aguda e grave relacionada à COVID19 em crianças e adolescentes, é importante ressaltar que essas ocorrências são raras até o momento. Entretanto, o avançar do conhecimento da patogenia dessa doença possibilitará terapias mais específicas possibilitando um melhor prognóstico.

\section{REFERÊNCIAS}

CAMPOS, Leonardo Rodrigues et al. Síndrome inflamatória multissistêmica pediátrica (MIS-C) temporariamente associada ao SARS-CoV-2. Residência Pediatria, 2020. DOI:10.25060/residpediatr-2020.v10n2-348. Disponível em:< https://cdn.publisher.gn1.link/residenciapediatrica.com.br/pdf/rp300720a03.pdf >. Acesso em 28 de agosto de 2020.

CDC, Centers for Disease Prevention and Control. Multisystem inflammatory syndrome in children (MIS-C) associated with coronavirus disease 2019 (COVID-19). Disponível em:<https://emergency.cdc.gov/han/2020/han00432.asp>. Acesso em 28 de agosto de 2020.

MCCRINDLE, Brian W. et al. Diagnosis, Treatment, and Long-Term Management of Kawasaki Disease: a scientific statement for health professionals from the american heart association. Circulation, [S.L.], v. 135, n. 17, p. 1-73, 25 abr. 2017. Ovid Technologies (Wolters Kluwer Health). DOI: http://dx.doi.org/10.1161/cir.0000000000000484. Disponível em: <https://www.ahajournals.org/doi/epub/10.1161/CIR.0000000000000484>. Acesso em 10 de setembro de 2020.

PANUPATTANAPONG, Sirada; BROOKS, Elizabeth B. New spectrum of COVID-19 manifestations in children: kawasakilike syndrome and hyperinflammatory response. Cleveland Clinic Journal Of Medicine, [S.L.], p. 1-4, 3 jun. 2020. Cleveland Clinic Journal of Medicine. DOI: http://dx.doi.org/10.3949/ccjm.87a.ccc039. Disponível em:< https://www.ccjm.org/content/early/2020/06/01/ccjm.87a.ccc039>. Acesso em 13 de setembro de 2020. RAMCHARAN, Tristan et al. Paediatric Inflammatory Multisystem Syndrome: temporally associated with sars-cov-2 (pims-ts). Pediatric Cardiology, [S.L.], p. 1-11, 12 jun. 2020. Springer Science and Business Media LLC. http://dx.doi.org/10.1007/s00246-020-02391-2. Disponível em: $<$ https://www.ncbi.nlm.nih.gov/pmc/articles/PMC7289638/>. Acesso em 09 de setembro de 2020. ROYAL COLLEGE OF PAEDIATRICS AND CHILD HEALTH (RCPCH). Guidance: Paediatric multisystem inflammatory syndrome temporally associated with COVID-19 (PIMS). 2020. Disponível em: < https://www.rcpch.ac.uk/resources/guidance-paediatric-multisystem inflammatory-syndrome-temporallyassociated-covid-19-pims>. Acesso em: 8 de setembro de 2020.

SOCIEDADE BRASILEIRA DE PEDIATRIA. Nota de alerta: Notificação obrigatória no Ministério da Saúde dos casos de síndrome inflamatória multissistêmica pediátrica (SIM-P) potencialmente associada à COVID-19. Sociedade Brasileira de Pediatria, [S. I.], p. 4-6, 7 ago.2020. Disponível em:<https://www.sbp.com.br/fileadmin/user_upload/22682bNA_NotificacaoObrigatoria_no_MS_dos_SIM-Covid19.pdf>. Acesso em 8 de setembro de 2020.

SOCIEDADE BRASILEIRA DE PEDIATRIA EM SÃO PAULO. Síndrome inflamatória multissistêmica pediátrica (síndrome associada temporalmente ao COVID-19). São Paulo, 2020. Disponível em:<

https://www.spsp.org.br/2020/05/19/sindrome-inflamatoria-multissistemica-pediatrica/>. Acesso em 28 de agosto de 2020.

SOCIEDADE BRASILEIRA DE REUMATOLOGIA (SBR). Nota de alerta sobre síndrome inflamatória multissistêmica em crianças e adolescentes associada à COVID-19 [Internet]. Disponível em:

<https://www.reumatologia.org.br/notícias>. Acesso em 28 de agosto de 2020.

VINER, Russell. WHITTAKER, Elizabeth. Kawasaki-like disease: emerging complication during the COVID-19 pandemic. Lancet. DOI: https://doi.org/10.1016/S0140-6736(20)31129-6. Disponível em:< https://www.ncbi.nlm.nih.gov/pmc/articles/PMC7220168/>. Acesso em 8 de setembro de 2020.

WHO. Word Health Organization. Multisysteminflammatorysyndrome in children and adolescents temporally related to COVID-19. [S. I.], 2020. Disponível em: < https://www.who.int/news-room/commentaries/detail/multisysteminflammatory-syndrome-in-children-and-adolescents-with-covid-19>. Acesso em 8 de setembro de 2020. 